\title{
Effects of RegCM3 parameterizations on simulated rainy season over South America
}

\author{
Rosmeri Porfirio da Rocha ${ }^{1, *}$, Santiago Vianna Cuadra ${ }^{2}$, Michelle Simões Reboita ${ }^{3}$, \\ Luiz F. Kruger ${ }^{1}$, Tércio Ambrizzi ${ }^{1}$, Nisia Krusche ${ }^{4}$ \\ ${ }^{1}$ Department of Atmospheric Sciences, University of São Paulo, Rua do Matão, 1226, Cid. Universitária, São Paulo, \\ SP 05508-090, Brazil \\ ${ }^{2}$ Department of Atmospheric Sciences, Federal Center of Technological Education Celso Suckow da Fonseca (CEFET/RJ), \\ Av. Maracanã, 229, Rio de Janeiro, RJ 20271-110, Brazil \\ ${ }^{3}$ Environmental Resource Institute, Federal University of Itajubá (UNIFEI), Av. BPS, 1303, Itajubá, MG 37500-903, Brazil \\ ${ }^{4}$ Computer Science Center, Federal University of Rio Grande, Av. Itália, km 8, Rio Grande, RS 96201-900, Brazil
}

\begin{abstract}
The impacts of change in the Grell convective scheme and biosphere-atmosphere transfer scheme (BATS) in RegCM3 are described. Three numerical experiments (RegZhang, RegClaris and RegArain) are conducted to reduce the RegCM3-Grell rainfall underestimation over tropical South America. The simulation referred to as RegZhang follows modifications made by Zhang et al. (2008) in the BATS. The RegClaris combines the RegZhang BATS parameters with a reduction of water drainage at the bottom of the subsoil layer in the regions covered by the tropical rain forest and a shorter convective time period for the Grell scheme. The RegArain considers this same modification in the Grell scheme, but uses a deeper total soil column and a deeper root system in the BATS. After the first year of simulation, the soil water content in RegZhang is progressively drained out of the soil column resulting in a deficit of rainfall in the Amazon. The RegClaris and RegArain, on the other hand, simulate a similar rainfall annual cycle in the Amazon, showing substantial improvement not only in phase but also in intensity. This improvement is partially related to an increase in evapotranspiration due to a larger availability of water in the soil column. A remote effect is also noted over the La Plata Basin region, where the larger summer rainfall rate may be related to the increase in moisture transport from the Amazon. Wind- and rainfall-based indices are applied to identify South American monsoon (SAM) timing. The RegClaris rainfall rates are adequate to identify the onset and the demise of SAM according to the observed data, whereas the rainfall deficit in RegZhang is associated with a delay in the onset and an early demise of the SAM.
\end{abstract}

KEY WORDS: Regional climate model - Annual cycle of precipitation · South American monsoon · Land-surface model · Amazon Basin hydrology

\section{INTRODUCTION}

The possibility of improving global model results using regional climate models (RCMs) began to be investigated in the 1990s (Dickinson et al. 1989, Giorgi \& Marinucci 1991), with a main focus on North America and Europe. At the moment, RCMs are largely used to downscale analyses of observed data (reanalysis) and outputs of global models (for seasonal forecasting, climate change studies, physical analysis of climate, etc.), and they are applied on many parts of the globe. Giorgi \& Mearns (1999) emphasized that the use of RCMs is mostly focused on obtaining a better representation of rainfall and near- 
surface variables (air temperature, relative humidity, winds, etc.) that may improve the representation of local climate.

In particular, for South America (SA), many recent studies have investigated the potential of using different RCMs (Eta, MM5, RSM, RegCM3 and HadRM3) to better understand present day climate (Chou et al. 2000, Nicolini et al. 2002, Nunes \& Roads 2005, Fernandez et al. 2006, Seth et al. 2007, da Rocha et al. 2009a) and to explore future climate scenarios (Nuñes et al. 2009, Marengo et al. 2010). In these simulations the horizontal resolution ranged from $120 \mathrm{~km}$ (Nicolini et al. 2002) to $40 \mathrm{~km}$ (Chou et al. 2000), focusing on the reproduction of the mean climate and its interannual and intraseasonal variability.

Simulations show that the Regional Climate Model Version 3 (RegCM3; Pal et al. 2007) using Grell's (Grell 1993) convective scheme generally underestimates rainfall in the SA tropical domain (Fernandez et al. 2006, Cuadra \& da Rocha 2007, Rauscher et al. 2007, Seth et al. 2007, da Rocha et al. 2009a). This dry bias usually occurs during the summer in the Amazon and South Atlantic Convergence Zone (SACZ; Kodama 1992). The SACZ is the main precipitation system in SA, usually producing intense and/or persistent rainfall over extensive areas in the tropics and subtropics (Carvalho et al. 2002). The rainfall underestimation is particularly evident in the simulations performed by Seth et al. (2007), where the precipitation and root layer soil moisture simulated for the Amazon region were very low.

Although underestimating the rainfall intensity over the tropical and subtropical SA regions, da Rocha et al. (2009a) showed that RegCM3 using Grell's convective scheme simulated most features of the observed diurnal cycle of precipitation. This indicates the potential of applying this RegCM3 configuration to study precipitation systems over SA, but it is necessary to reduce the dry bias. Many application areas (agricultural, hydrological, health, etc.) need the best possible information about the amounts of precipitation and its high-frequency variability. For example, $5 \mathrm{~d}$ average precipitation intensity is often used to define the onset and end of the rainy season (Liebmann \& Marengo 2001, Gan et al. 2005, Rauscher et al. 2007), which is important information for agricultural applications.

The present paper explores parameter changes in the biosphere-atmosphere transfer scheme (BATS; Dickinson et al. 1993) and Grell's convective schemes of RegCM3 in order to improve predictions of the rainfall intensity over tropical SA. The impacts of these changes in the climatology and timing of the rainy season are also discussed. RegCM3 simulations were performed within the context of the CLARISLPB (a Europe-South America network for climate change assessment and impact studies in La Plata Basin) and CORDEX (coordinated regional downscaling experiment; Giorgi et al. 2009) projects.

\section{DATA AND METHODOLOGY}

\subsection{Data}

In 2007, the European Centre for Medium-Range Weather Forecasts (ECMWF) made available the ERA-Interim reanalysis, which is a more extensive data set than the ERA-40 (Simmons et al. 2007). The ERA-Interim archive has a greater number of vertical pressure levels (37), added cloud parameters, and has a higher horizontal resolution $\left(1.5^{\circ}\right)$ than ERA-40.

We compare RegCM3 precipitation simulation with 2 data sets: the monthly climatology of the Climate Prediction Center merged analysis of precipitation (CMAP; Xie \& Arkin, 1996) and the daily precipitation analysis from the Climate Prediction Center $\left(\mathrm{CPC}_{i}\right.$ Silva et al. 2007). The CMAP includes the product of global satellite and rain gauge data, and it is available on a $2.5^{\circ}$ latitude-longitude grid. The CPC analysis considers only rain gauge data and has $1.0^{\circ}$ of horizontal resolution. The daily rainfall from $\mathrm{CPC}$ is used to calculate the pentads in order to analyze rainy season timing.

Simulations are also compared against monthly averaged micrometeorological measurements of 3 experimental sites over the Amazon rainforest. The sites are referred to as Manaus KM34 $\left(2.6090^{\circ} \mathrm{S}\right.$, $\left.60.2093^{\circ} \mathrm{W}\right)$, Santarem KM67 $\left(2.8853^{\circ} \mathrm{S}, 54.9205^{\circ} \mathrm{W}\right)$ and Santarem KM83 $\left(3.0502^{\circ} \mathrm{S}, 54.9280^{\circ} \mathrm{W}\right)$, and their locations are shown in Fig. 1. Observed precipitation, net radiation $\left(R_{\mathrm{n}}\right)$, and sensible $(H)$ and latent $(\lambda E)$ heat fluxes are used to evaluate the simulated surface energy budget. The $H$ and $\lambda E$ fluxes are derived from the eddy correlation technique, and a summary of these micrometeorological observations can be obtained in Negrón-Juárez et al. (2007). The climatologies of several variables observed at the sites are shown in da Rocha et al. (2009b).

\subsection{Atmospheric model}

As documented by Pal et al. (2007), the RegCM3 solves the equations for a compressible atmosphere in finite differences, considering the hydrostatic ap- 


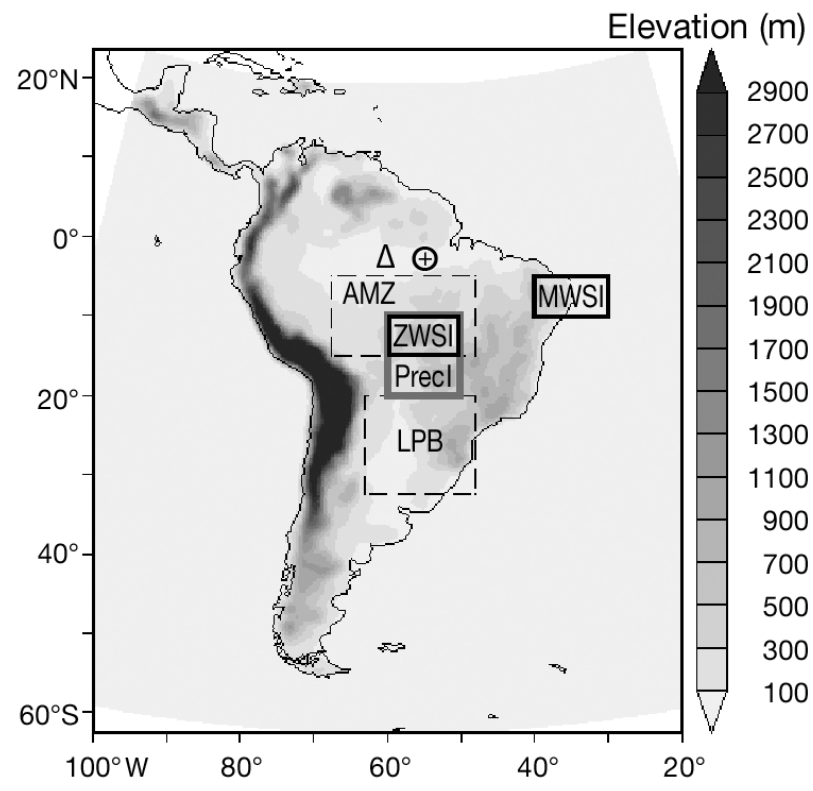

Fig. 1. Domain, topography and location of the Amazon (AMZ) and La Plata Basins (LPB) (dashed boxes). The figure also includes the location of the regions used in the calculation of the onset and demise indexes of the South American monsoon: PrecI $\left(20-10^{\circ} \mathrm{S}, 60-50^{\circ} \mathrm{W}\right)$, ZWSI $\left(15-10^{\circ} \mathrm{S}\right.$, $\left.60-50^{\circ} \mathrm{W}\right)$ and MWSI $\left(10-5^{\circ} \mathrm{S}, 40-30^{\circ} \mathrm{W}\right)$ and the locations of the LBA sites Manaus KM34 (triangle), Santarem KM67 (open circle) and Santarem KM83 (cross)

proximation and a sigma-pressure vertical coordinate. The radiative processes in this model are calculated as in the Community Climate Model 3 (CCM3; Kiehl et al. 1996) parameterization. The heating rate and surface fluxes for solar and infrared radiation are computed separately considering clear skies and cloudy conditions. The effect of greenhouse gases $\left(\mathrm{NO}_{2}, \mathrm{CH}_{4}\right.$ and chlorofluorocarbons), atmospheric aerosols and cloud water are also included in the CCM3 radiation parameterization.

The moist processes in the RegCM3 are parameterized considering 1 convective scheme for deep cumulus convection and 1 grid scale precipitation scheme. The latter was developed by Pal et al. (2000) and is a simplified scheme that solves only the prognostic equation for cloud water, which is then directly used in radiative transfer parameterization. The Grell convective scheme is a simplified version of the Arakawa \& Schubert (1974) parameterization. The RegCM3 has 2 closure assumptions that can be used in the Grell scheme (Pal et al. 2007). The ArakawaSchubert closure assumes that the large-scale destabilization processes are in quasi-equilibrium with the convection. In the Fritsch-Chappell (FC) closure all the convective available potential energy (CAPE) is dissipated during a specified convective time period, with a default value of $30 \mathrm{~min}$. The present study uses the FC closure and tests the convective time period.

The land-surface physics in the RegCM3 is solved by BATS, a land-surface model that simulates the interactions between vegetation, soil system and atmosphere, taking into account the turbulent moment, energy and water vapor exchange between the surface and atmosphere. The BATS scheme considers 1 vegetation layer (20 vegetation types [VT] in RegCM3) and 3 soil layers. The rooting ratios and upper and total soil depths are functions of landcover type; therefore, each VT has its corresponding soil properties. For instance, the default rooting zone (ranging from 1.0 to $2.0 \mathrm{~m}$ ), depends on the VT. All VTs have a total soil depth of $3 \mathrm{~m}$ to represent ground water storage.

\subsection{Simulation setup}

The simulation domain was defined following the CORDEX, an international effort to downscale climate projections over the world using RCMs (Giorgi et al. 2009). The simulations used the Mercatorrotated projection, with a $50 \mathrm{~km}$ horizontal resolution-192 $\times 202$ grid points in east-west and north-south directions, respectively-and, vertically, 18 sigma-pressure levels. Fig. 1 shows the domain, topography and areas of the Amazon (AMZ) and La Plata Basins (LPB) and the regions used in the rainy season analysis. The simulations are driven by atmospheric variables (geopotential height, temperature, wind, relative humidity and pressure) and sea-surface temperature (SST) from ERA-Interim, integrated from 1 January 1989 to 1 January 2009.

\subsection{Indices to identify onset and demise of the South American monsoon}

The onset and demise of the South American monsoon (SAM) simulated by the RegCM3 and obtained from the ERA-Interim data are analyzed using 3 indices suggested by Gan et al. (2005 - hereafter called GAN), and defined as follows:

Precipitation index (PrecI): this index is defined considering the pentad mean precipitation over West-Central Brazil region $\left(10^{\circ}\right.$ to $20^{\circ} \mathrm{S}, 50^{\circ}$ to $60^{\circ} \mathrm{W}$; see Fig. 1). The SAM onset (demise) occurs when a pentad shows precipitation higher (lower) than 
$4 \mathrm{~mm} \mathrm{~d}^{-1}$, and this value persists during at least 6 out of 8 subsequent pentads.

Zonal wind shear index (ZWSI): the 850 and $200 \mathrm{hPa}$ zonal wind pentad averages over WestCentral Brazil region $\left(10^{\circ}\right.$ to $15^{\circ} \mathrm{S}, 50^{\circ}$ to $60^{\circ} \mathrm{W}$; see Fig. 1) is calculated, and the vertical shear (200 to $850 \mathrm{hPa}$ ) is determined. The SAM onset (demise) occurs when a pentad presents vertical shear lower (higher) than $8 \mathrm{~m} \mathrm{~s}^{-1}$, and this feature prevails during at least 6 out of 8 subsequent pentads. Negative ZWSI indicates that the Bolivian High - a system that characterizes SA austral summer and SAM establishment (Gandu \& Geisler 1991, Lenters \& Cook 1997) - is active. The Bolivian High is characterized by a large anticyclonic circulation at $200 \mathrm{hPa}$ centered over Bolivia $\left(\sim 17^{\circ} \mathrm{S}, 63^{\circ} \mathrm{W}\right)$ and is the result of the $\lambda E$ release due to strong summer convective activity in the Amazon Basin (Figueroa et al. 1995, Lenters \& Cook 1997).

Meridional wind shear index (MWSI): vertical wind shear, between 200 and $850 \mathrm{hPa}$, is calculated for pentad mean meridional wind over northeastern Brazil $\left(5^{\circ}\right.$ to $10^{\circ} \mathrm{S}, 30^{\circ}$ to $40^{\circ} \mathrm{W}$; see Fig. 1$)$. The monsoon onset (demise) occurs when a pentad presents positive (negative) vertical shear, and this feature is maintained during 6 out of 8 subsequent pentads. A positive MWSI indicates the presence of a trough over Northeast Brazil, with predominantly southerly winds in the MWSI area (Fig. 1), which is usually observed downstream of the Bolivian High during SAM (Lenters \& Cook 1997).

For the 1979-1997 period the GAN comparison of indices based on wind and rainfall showed that there is a high correlation between the ZWSI and MWSI and the PrecI index. However, they also noted that MWSI tends to indicate a delayed SAM onset when compared to the ZWSI index.

\section{RESULTS}

\subsection{Testing parameters in the BATS and Grell scheme}

Previous investigations of summer rainfall simulated by the RegCM3 with Grell's convective scheme over SA generally reported dry biases over the AMZ and LPB regions (Seth et al. 2007, da Rocha et al. 2009a, da Silva et al. 2010). To overcome this rainfall underestimation over the $\mathrm{AMZ}$, which may also affect the moisture transport and rainfall over the LPB (see Vera et al. 2006), changes in the BATS land-surface model and Grell's convective scheme were tested.
Initially, changes in BATS followed the suggestions reported by Zhang et al. (2008), from now on referred to as Zhang. Zhang modified BATS parameters over the tropical rain forest (VT 6 in the BATS scheme) trying to decrease/increase the $H / \lambda E$ fluxes over the AMZ. They changed the ratio of root distribution between the upper soil layer $(0.1 \mathrm{~m})$ and the root zone layer $(3.0 \mathrm{~m})$ from 0.8 to 0.4 and changed the BATS default root zone layer and total soil depths from 1.5 and $3.0 \mathrm{~m}$ to 3.0 and $4.5 \mathrm{~m}$, respectively. The RegCM3 simulation with Zhang soil and root property modifications for the tropical rain forest is referred to as RegZhang (Table 1).

However, as previously noted by Zhang, their implementations were not able to sustain soil water content to support a realistic daily maximum evapotranspiration (ET). Consequently, precipitation is insufficient to maintain the soil water content at reasonable levels, and ET is excessively low during most of the year in the RegCM3 simulations when BATS and Grell convective parameterizations are used. This feature is also evident in Fig. 9b in Seth et al. (2007), obtained with these parameterizations, where the 400 to $420 \mathrm{~mm}$ of root soil moisture is less than half of that typically observed in the Amazon Basin (e.g. Negrón-Juárez et al. 2007). One possible hypothesis to explain such low soil water content simulated by BATS is that water flows too quickly through and out of the soil column, implying excessive subsoil drainage. It is important to note that some mechanisms to maintain moistened near-surface soil, i.e. increasing the amount of water accessible to the root system, are neglected in the BATS model. For example, the impacts of shallow water and hydraulic redistribution are not considered in BATS (e.g. da Rocha et al. 2004, Oliveira et al. 2005, Fan \& Miguez-Macho 2010). Another possible way to better adjust the

Table 1. Values of the modified parameters in the biosphereatmosphere transfer scheme for tropical rain forest (evergreen broadleaf tree, Vegetation Type 6) and for Grell's convective scheme over the whole domain. The variable/parameter names in RegCM3 code are in italics; deptv: depth of the total soil; deptrv: depth of the root layer; rootf: ratio of root distribution between the upper soil layer and root zone soil layer; $x k m \times 2$ : saturated hydraulic conductivity at the bottom of the subsoil layer; dtauc: Grell convective time period

\begin{tabular}{|lccccc|}
\hline Simulation & $\begin{array}{c}\text { deptv } \\
(\mathrm{m})\end{array}$ & $\begin{array}{c}\text { deptrv } \\
(\mathrm{m})\end{array}$ & rootf & $\begin{array}{c}\mathrm{xkmx2} \\
\left(\mathrm{mm} \mathrm{s}^{-1}\right)\end{array}$ & $\begin{array}{c}\text { dtauc } \\
(\mathrm{min})\end{array}$ \\
\hline Default & 3.0 & 1.5 & 0.8 & $4.0 \times 10^{-4}$ & 30 \\
RegZhang & 4.5 & 3.0 & 0.4 & $4.0 \times 10^{-4}$ & 30 \\
RegClaris & 4.5 & 3.0 & 0.4 & $1.6 \times 10^{-4}$ & 25 \\
RegArain & 8.0 & 4.0 & 0.2 & $4.0 \times 10^{-4}$ & 25 \\
\hline
\end{tabular}


default configuration and to overcome the lack of such mechanisms in BATS is to deepen the total soil column and root system, increasing the total water storage capacity and the fraction available to the root system (e.g. Arain et al. 1997, Baker et al. 2008), according to observational evidence for deep rooting in the Amazon Basin (e.g. Nepstad et al. 1994).

We explore the impacts of higher soil water content through 2 new RegCM3 experiments with additional changes in BATS and Grell's convective schemes (Table 1). The BATS parameters are only modified for the tropical rain forest (evergreen broadleaf tree, VT 6), while the parameter change in the Grell scheme affects the whole domain. For the RegClaris simulation, the saturated hydraulic conductivity at the bottom of the subsoil layer was defined as $40 \%$ of its default value (in addition to the previous changes made in RegZhang), therefore reducing the soil water drainage. The applied value was obtained through sensitivity analysis. In the simulation referred to as RegArain, the ratio of the root distribution between the upper soil layer $(0.1 \mathrm{~m})$ and the root zone layer $(4.0 \mathrm{~m})$ was changed from 0.8 to 0.2 , and the total soil column and root zone layer were deepened to 8.0 and $4.0 \mathrm{~m}$, respectively, following the suggestion of Arain et al. (1997).

Additionally, the Grell-FC convective time period is changed from 30 (default) to $25 \mathrm{~min}$ in the RegClaris and RegArain simulations. This modification reduces the necessary time for the convective clouds to remove the available potential energy for convection. Some analyses using RegCM3-Grell-FC over SA (not shown) suggest its sensitivity to the convective time period, and this modification may help to increase the amount of convective precipitation. Table 1 summarizes the modifications for the RegZhang, RegClaris and RegArain simulations.

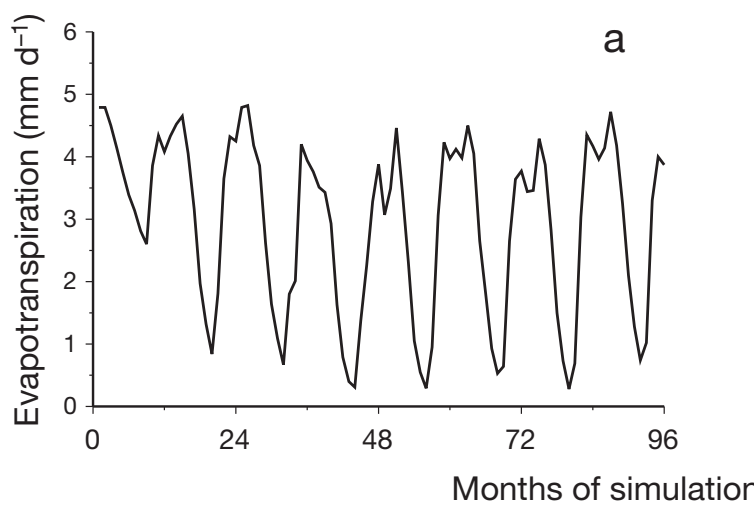

Fig. 2. Monthly time series of (1 = January 1989): (a) RegZhang evapotranspiration (mm $\mathrm{d}^{-1}$ ) and (b) RegZhang root zone soil

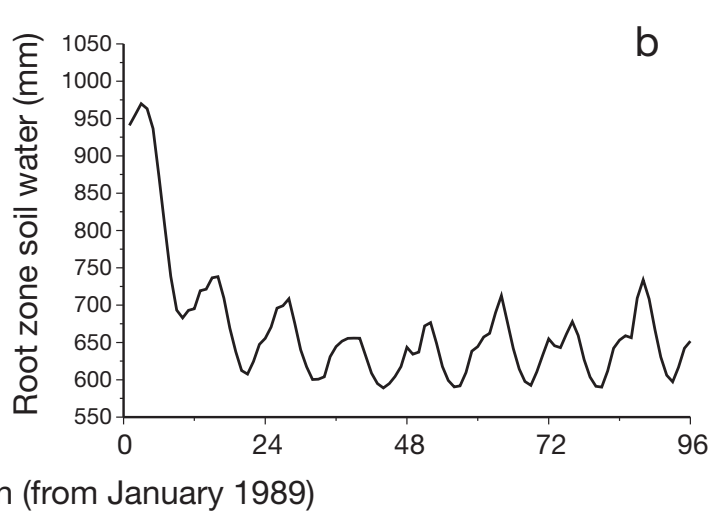

By applying Zhang changes, an increase in the $\lambda E$ flux and a reduction in the $H$ flux - yielding a Bowen ratio $(\beta ; H: \lambda E)$ closer to the measurements — was only maintained in the first year of simulation. After that, there was a large rainfall underestimation during summer and an increase in the ET annual cycle amplitude (ranging from 0.5 to $4.5 \mathrm{~mm} \mathrm{~d}^{-1}$ ), i.e. a very low ET during the dry season (Fig. 2a). Fig. 2b reveals that soil water in the root zone in the first months of simulation presented an average value of $\sim 950 \mathrm{~mm}$, which is similar to the value reported by Zhang. However, Fig. 2b also shows that after the first year of simulation, the root zone soil water content decreases to $\sim 650 \mathrm{~mm}$, a value lower than the one previously reported by Zhang and close to those shown by Seth et al. (2007).

Fig. 3a,b shows the ET and root zone soil water annual cycles obtained from the 3 simulations. The total water content of the soil root zone in the RegClaris and RegArain simulations are closer to the observed values for the AMZ region (e.g. Hodnett et al. 1995, Negrón-Juárez et al. 2007), while the values simulated by RegZhang ( 650 mm; Fig. 3b) are extremely low when compared to the observations. The difference between the total soil root zone water in the RegClaris and RegArain (Fig. 3b) comes mostly from the difference in the depth of this layer ( 3 and $4 \mathrm{~m}$, respectively) - their average volumetric soil water contents are quite similar. Additionally, the ET (Fig. 3a) and precipitation (Section 3.2) simulated in these experiments are also similar, suggesting that the parameter changes generate almost identical surface heat fluxes and interactions with the atmosphere. Therefore, since RegClaris and RegArain have produced similar atmospheric conditions, the following analyses will only compare RegZhang and RegClaris simulations.

\footnotetext{
water content (mm)
} 

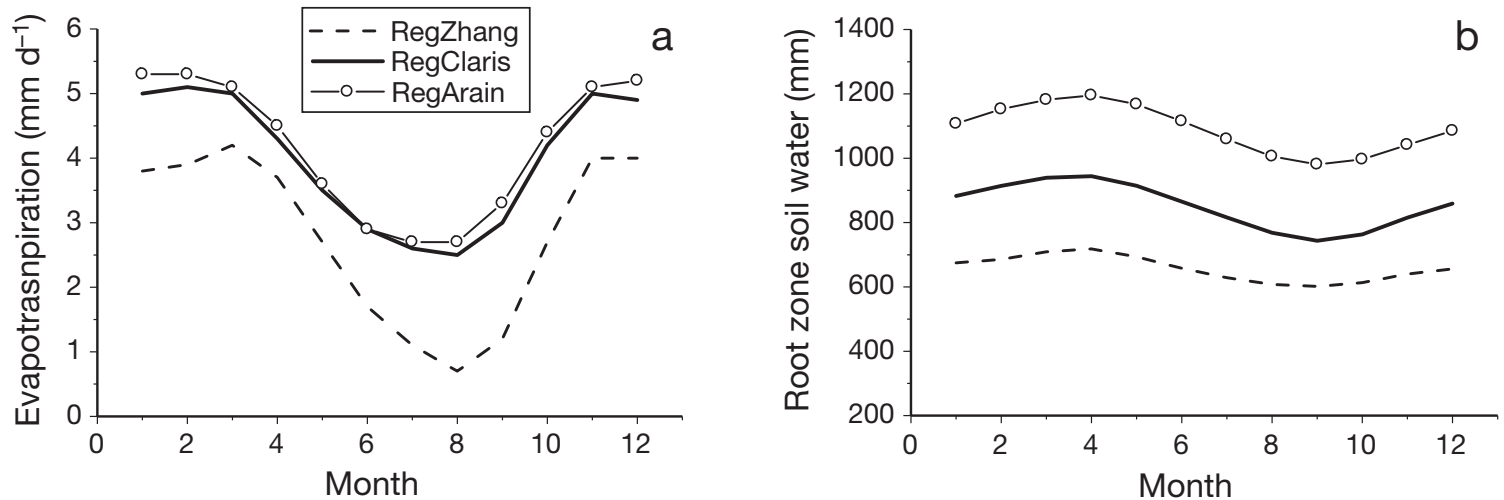

Fig. 3. Annual cycles of: (a) evapotranspiration $\left(\mathrm{mm} \mathrm{d}^{-1}\right)$ and (b) root zone soil water (mm) as obtained from RegZhang, RegArain and RegClaris in the Amazon region noted in Fig. 1

To evaluate the impacts of parameter changes in the surface energy budget, the simulations were compared with data from 3 observational sites (Manaus KM34, Santarem KM67 and Santarem KM83), for
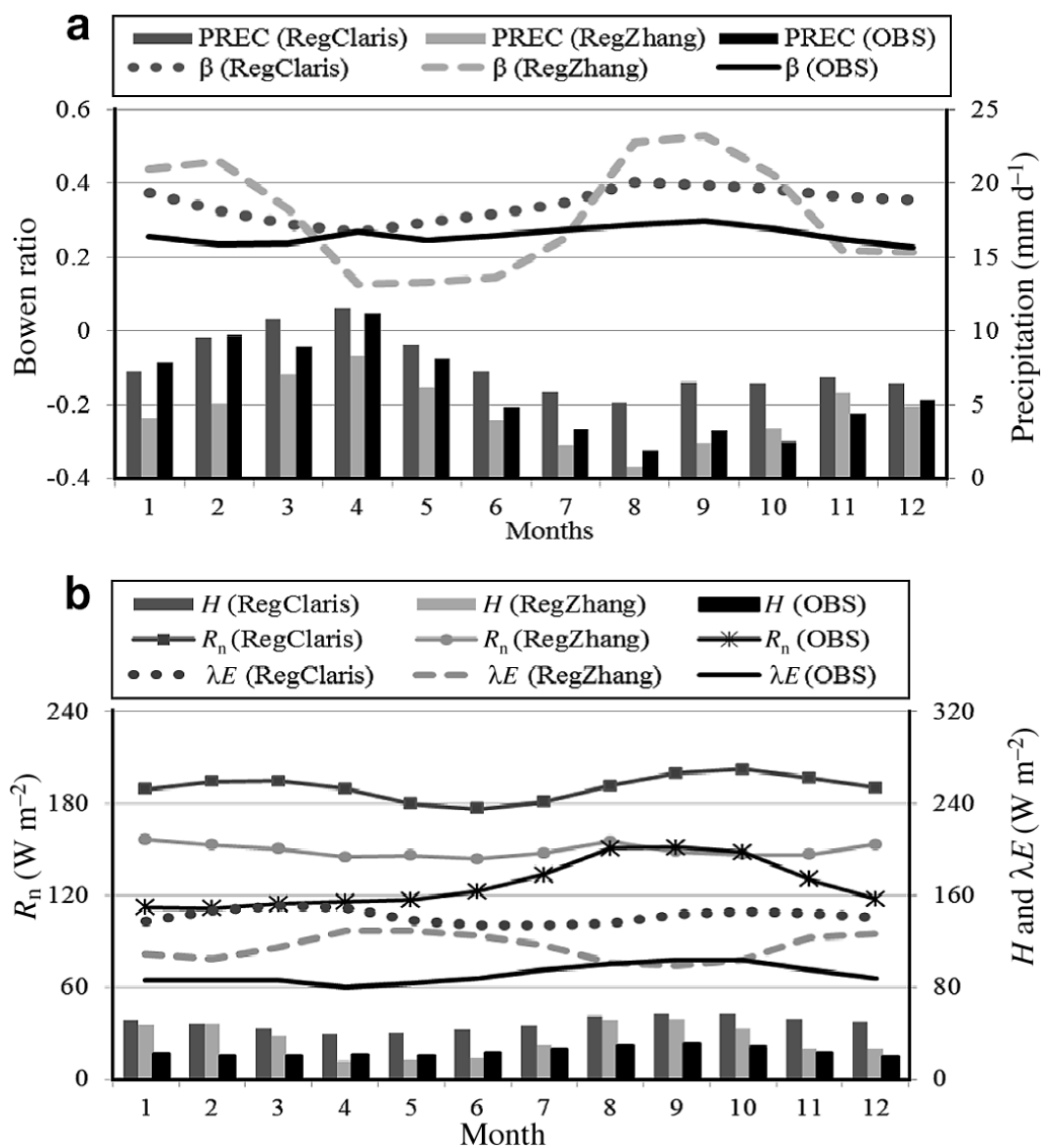

Fig. 4. (a) Observed and simulated (RegClaris and RegZhang) precipitation and Bowen ratio ( $\beta)$. (b) Observed and simulated net radiation $\left(R_{\mathrm{n}}\right)$, sensible heat flux $(H)$, and latent heat flux $(\lambda E)$. All values consider the average for the 3 sites: Manaus KM34, Santarem KM83 and Santarem KM67 for the period 1998-2006 the period 1998-2006 (Fig. 4). From Fig. 4a, it is clear that the Bowen ratio $(\beta)$ is substantially improved in RegClaris, particularly the annual cycle pattern. In general, the precipitation rate simulated by RegClaris is similar to the observational sites. Although the partition between $\lambda E$ and $H$ improved in RegClaris, Fig. $4 \mathrm{~b}$ indicates an increase in $R_{\mathrm{n}}$, and consequently an overestimation of the $H$ and $\lambda E$ fluxes. Nevertheless, compared with local observations, RegClaris improves the $H$ and $\lambda E$ annual cycles in terms of phase and amplitude. The higher $R_{\mathrm{n}}$ in RegClaris (and RegArain) simulation is probably due to a higher precipitation rate and a decrease in cloud cover and specific humidity. For example, at an annual scale there is a reduction of $\sim 7 \%$ in the total cloud cover over the AMZ region, and, consequently, an increase in the incident solar radiation at the surface.

\subsection{Precipitation climatology and annual cycle}

Fig. 5 shows austral summer (December, January and February, DJF) and winter (June, July and August, JJA) rainfall climatologies obtained from the CMAP analysis and simulated by RegClaris and RegZhang. The RegClaris summer rainfall (Fig. 5c) has a pattern that is more similar to CMAP (Fig. 5a) than to RegZhang (Fig. 5b). 


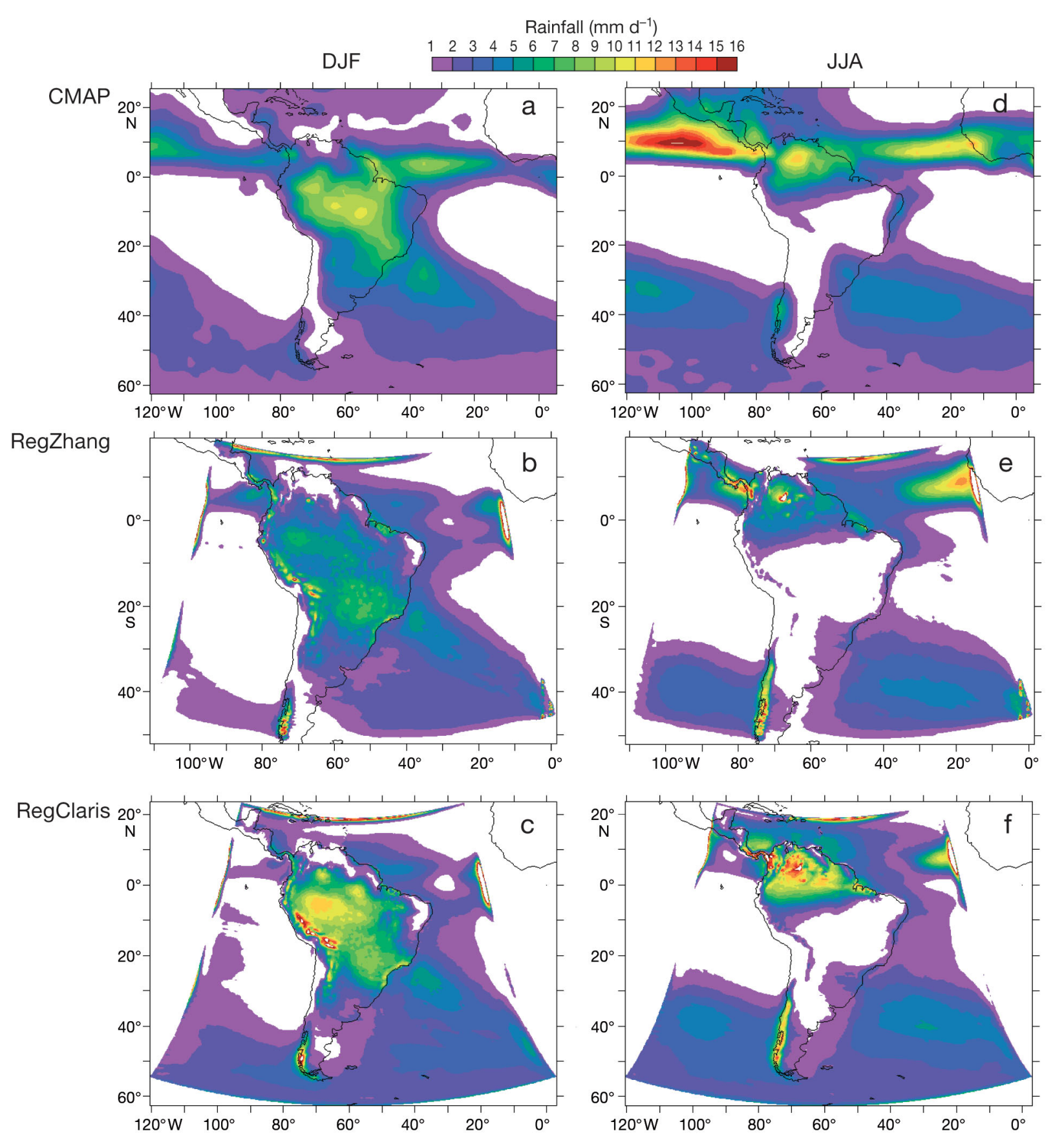

Fig. 5. Simulated and observed rainfall in austral summer (December, January and February, DJF, left) and winter (June, July and August, JJA, right), 1989-2008, from: (a,d) CMAP, (b,e) RegZhang and (c,f) RegClaris

The rainfall intensification over the AMZ region may be partially attributed to large ET in the RegClaris (Fig. 3a) and its impacts on atmospheric stability and water content. In terms of stability, higher ET increases the atmospheric moist static energy and, consequently, the triggering of the Grell convective scheme. Therefore, the reduction of deep water percolation implies a positive feedback of water maintenance in the soil column (Fig. 3b), and rainfall increases during the year in the RegClaris simulation. Fig. 5a-c also shows that the RegClaris rainfall rate over the southeast and oceanic branches of the SACZ is similar to that in the CMAP analysis, while RegZhang largely underestimates the precipitation over this area (Fig. 5b).

During the winter season (Fig. $5 \mathrm{~d}-\mathrm{f}$ ), the displacement of the Amazon Basin rainfall to the north indicated in the CMAP field is well simulated by the 
model. In the RegClaris the intense precipitation is spread over a larger area over northern SA, while the RegZhang underestimates the rainfall in this area. Therefore, as in summer, the tropical winter rainfall simulated by RegClaris also has a pattern more consistent with that of CMAP than with that of the RegZhang simulation. Additionally, RegClaris (Fig. 5f) shows a large area with rainfall values $>2 \mathrm{~mm} \mathrm{~d}^{-1}$ centered over $30^{\circ} \mathrm{S}, 50^{\circ} \mathrm{W}$ (southern Brazil), which is in agreement with the CMAP analysis (Fig. 5d).

The RegClaris (and RegArain) simulation shows a clear improvement in the rainfall annual cycle for the AMZ region, particularly a significant reduction in the dry bias during the rainy season (Fig. 6a). The RegClaris, RegArain, RegZhang and CMAP mean annual rainfall rates are 5.4, 5.4, 3.2 and $5.2 \mathrm{~mm} \mathrm{~d}^{-1}$, respectively. Figs. 3a \& 6a suggest a substantial influence of the land-surface system in the rainfall simulated over the AMZ region. Although the ET in RegZhang and RegClaris differ by only $\sim 1 \mathrm{~mm} \mathrm{~d}^{-1}$ during the wet season (Fig. 3a), the rainfall difference between these simulations is $\sim 3 \mathrm{~mm} \mathrm{~d}^{-1}$ (Fig. 6a). One possible mechanism to explain the increase in precipitation is through the positive feedback between the increase of $\lambda E$ release in the atmosphere, associated with larger amounts of precipitation, and the intensification of the SAM system (intensifying the low level convergence and upper level divergence). Additionally, Fig. $6 \mathrm{~b}$ suggests a remote effect in the rainfall annual cycle over the LPB, with a summer dry bias reduction in RegClaris (and RegArain). This may be associated with the increase in moisture transport from the AMZ to the LPB (data not shown), typical in the summer season. The annual rainfall in the LPB increases from $2.8 \mathrm{~mm}$ $\mathrm{d}^{-1}$ in RegZhang to $3.3 \mathrm{~mm} \mathrm{~d}^{-1}$ in the RegClaris and RegArain simulations, resulting in an improvement over the $3.8 \mathrm{~mm} \mathrm{~d}^{-1}$ of CMAP.

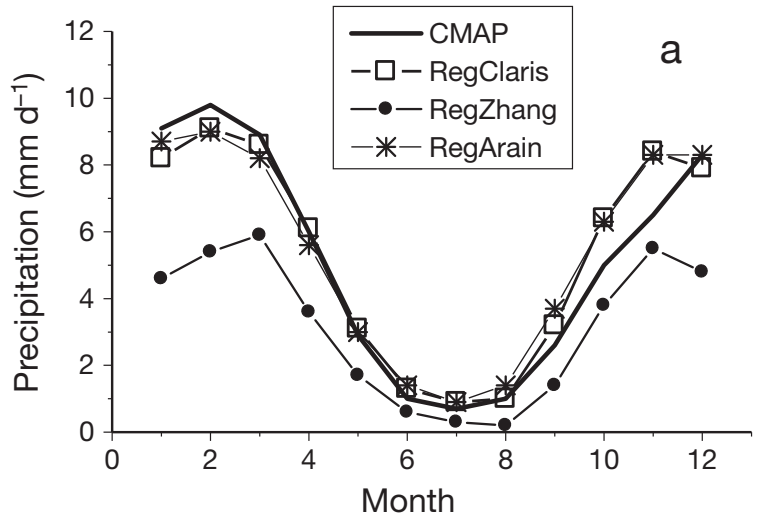

\subsection{Rainy season onset and demise}

An important question to be addressed is the increase of $\lambda E$ in the RegClaris experiment and its impact on the high frequency variability of precipitation (e.g. $5 \mathrm{~d}$ mean) and, consequently, in the drywet season transition over SA. Previous analyses by $\mathrm{Fu} \& \mathrm{Li}$ (2004) discussed the importance of surface processes to the timing of the SA rainy season. Using ECMWF reanalysis and local observations, Fu \& Li (2004) investigated the influence of surface $H$ and $\lambda E$ fluxes on the transition from the dry to wet season in southern Amazonia. According to these authors, this transition is initiated by an increase in surface $\lambda E$ flux and local precipitation, simultaneously with a reduction in $H$ fluxes. Thereafter, changes in local precipitation influence the large-scale circulation forcing the reversal of the cross-equatorial flow that produces large-scale moisture convergence over southern Amazonia. Similar sensitivity to the local $\lambda E$ fluxes for establishment of the rainy season was also discussed in Seth et al. (2011), but in the context of climate change scenarios.

The rainy season timing is addressed through SAM indices proposed by GAN. These indices were calculated using the RegClaris and RegZhang simulations (1989-2008), wind from ERA-Interim reanalysis (1989-2008) and CPC precipitation analysis (19892007). The results are compared with GAN who calculated the same indexes but using NCEP-NCAR (referred to as NCEP-NCAR/GAN) reanalysis and CPC rainfall (referred to as CPC/GAN) for the period 1979-1997.

When PrecI is considered, RegClaris simulates SAM onset and demise similar to the patterns derived from $\mathrm{CPC}$ and consistent to those from $\mathrm{CPC} /$ GAN (Table 2). On the other hand, RegZhang presents a delay of 3 and 2 pentads when compared to

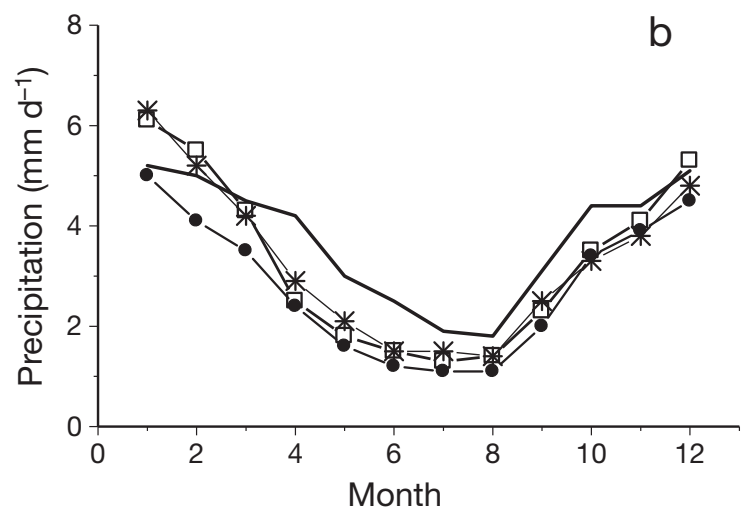

Fig. 6. Annual cycle of precipitation obtained from CMAP, RegClaris, RegArain and RegZhang for the (a) Amazon and (b) La Plata Basins 
CPC/GAN and CPC, respectively, for the SAM onset. With regards to SAM demise, RegZhang shows persistent earlier occurrence in comparison with CPC/ GAN (4 pentads earlier) and CPC analysis ( 3 pentads earlier). Therefore, RegZhang simulates a shorter rainy season than $\mathrm{CPC}$ analysis or CPC/GAN (Table 2). For RegClaris, the standard deviation of the onset date is similar to that of $\mathrm{CPC}$ and $\mathrm{CPC} /$ GAN, presenting a slightly larger variability for the demise of the SAM. For RegZhang, the standard deviations are larger than those of CPC for both onset and demise dates.

The better results of RegClaris in terms of SAM characteristics (onset, demise and length) can be explained through pentad mean precipitation. Over the PrecI area (see Fig. 1), Fig. 7a shows that the frequency distribution of rainfall pentads simulated by RegClaris presents large similarities with CPC analysis. For the period 1989-2007, the precipitation pentads have a correlation of 0.80 , indicating a large agreement between the 2 time series. Looking at the simulations, Fig. 7 a shows a reduction in the frequency of weaker rainfall events $\left(0-2 \mathrm{~mm} \mathrm{~d}^{-1}\right)$ and an increase in the frequency of more intense events $\left(>8 \mathrm{~mm} \mathrm{~d}^{-1}\right)$ in RegClaris compared to RegZhang. The larger frequency of weaker precipitation events can explain the delay in the onset of SAM simulated by RegZhang, as the PrecI index establishes a minimum of $4 \mathrm{~mm} \mathrm{~d}^{-1}$ and its persistence to identify the SAM onset and its maintenance.

Table 2. Pentad mean and standard deviation for the onset and demise of the South American monsoon as identified by the PrecI, ZWSI and MWSI obtained from Gan et al. (2005) (CPC/GAN and NCEP-NCAR/GAN), RegZhang, RegClaris, CPC analysis and ERA-Interim reanalysis. For more details on simulations see Section 2.4

\begin{tabular}{|c|c|c|}
\hline & Onset & Demise \\
\hline \multicolumn{3}{|l|}{ PrecI } \\
\hline CPC/GAN & $58 \pm 3$ & $22 \pm 2$ \\
\hline RegZhang & $61 \pm 4$ & $18 \pm 4$ \\
\hline RegClaris & $58 \pm 3$ & $20 \pm 3$ \\
\hline $\mathrm{CPC}$ & $59 \pm 3$ & $21 \pm 3$ \\
\hline \multicolumn{3}{|l|}{ ZWSI } \\
\hline NCEP-NCAR/GAN & $58 \pm 3$ & $23 \pm 2$ \\
\hline RegZhang & $54 \pm 3$ & $23 \pm 3$ \\
\hline RegClaris & $57 \pm 5$ & $22 \pm 4$ \\
\hline ERA-Interim & $58 \pm 4$ & $22 \pm 3$ \\
\hline \multicolumn{3}{|l|}{ MWSI } \\
\hline NCEP-NCAR/GAN & $62 \pm 5$ & $24 \pm 3$ \\
\hline RegZhang & $62 \pm 5$ & $21 \pm 5$ \\
\hline RegClaris & $57 \pm 6$ & $26 \pm 8$ \\
\hline ERA-Interim & $62 \pm 5$ & $23 \pm 8$ \\
\hline
\end{tabular}

Analyzing the timing of the rainy season over SA, Rauscher et al. (2007) found that the RegCM3 simulated the onset of the rainy season four pentads earlier in a region similar to that used in the PrecI index of the present work. From Table 2, it is clear that the RegClaris simulation of SAM timing has been substantially improved compared to the work of Rauscher et al. (2007).

Fig. 8 shows the time evolution of the composites of the $\lambda E$ and $H$ in the PrecI area (see Fig. 1). The $H$ increasing are similar for both RegZhang and RegClaris experiments until pentad -3 , before SAM onset, decreasing rapidly up to pentad +3 and showing stable behavior afterwards. $\lambda E$ begins to increase after pentad -12 and at the onset it jumps to higher values. As shown in Figs. $3 \& 4$, these fluxes are larger in RegClaris than in RegZhang during the year. Fig. 8 clearly shows the reversion of the flux tendencies after SAM onset. Our results are in agreement with those of $\mathrm{Fu} \& \mathrm{Li}$ (2004) who found a similar pattern in the southern Amazon Basin. As previously mentioned, intense $\lambda E$ after the onset may partially explain the larger precipitation rate and better definition of SAM timing in the RegClaris experiment.

As many atmospheric models have a deficiency in simulating the correct intensity of precipitation, GAN developed alternative wind-based SAM indices which are applied to the simulations (RegZhang and RegClaris) and ERA-Interim data sets. ZWSI is an index that considers the west-east circulation in the lower and upper levels. When the SAM onset (demise) begins over West-Central Brazil, zonal wind is westerly (easterly) at $850 \mathrm{hPa}$ and easterly (westerly) at $200 \mathrm{hPa}$ (e.g. Gan et al. 2004). For example, in the summer, easterly (negative value) wind at $200 \mathrm{hPa}$ is more intense (in module) than the westerly (positive value) wind at $850 \mathrm{hPa}$. This provides a negative ZWSI, and SAM onset is characterized by the persistence of this negative value in the next pentads.

By considering the ZWSI, Table 2 shows that RegClaris simulates SAM onset 1 pentad earlier than NCEP-NCAR/GAN and ERA-Interim. However, the date of SAM withdrawal is similar to that simulated with ERA-Interim and it occurs 1 pentad earlier than with NCEP-NCAR/GAN. The ZWSI results for the RegZhang simulation (Table 2) indicate that the SAM onset starts much earlier; however, the SAM demise is the same as with NCEP-NCAR/GAN. The interannual variabilities of the ZWSI obtained from ERAInterim and simulations are larger than those of NCEP-NCAR/GAN (Table 2).

The ZWSI frequency distributions for the RegClaris, RegZhang and ERA-Interim simulations are 

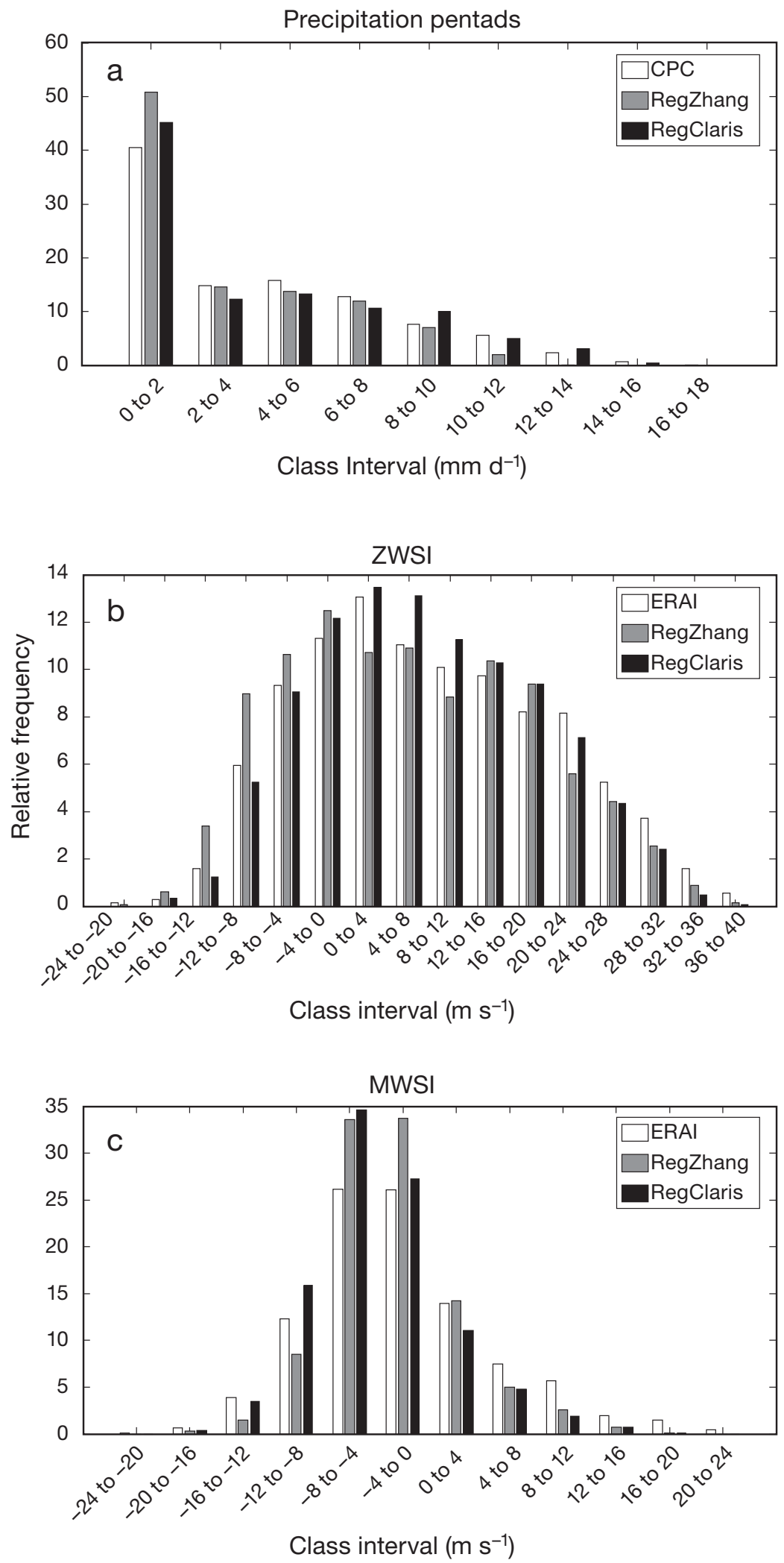

Fig. 7. Relative frequency histograms (1989-2007) of: (a) pentad mean precipitation, (b) ZWSI and (c) MWSI derived from CPC and ERA-Interim, RegZhang and RegClaris shown in Fig. 7b. RegClaris and RegZhang simulate ZWSI values highly correlated with ERA-Interim (0.87 and 0.90 , respectively). The frequency distributions of RegClaris and ERA-Interim show similar behavior for negative ZWSI values, which are used to detect SAM onset. This may explain the better result of the RegClaris compared to the RegZhang for the onset. In fact, RegZang overestimates the frequency of negative ZWSI values, which therefore influences the date of the start of the SAM (Table 2). Fig. 7b also suggests that the greater frequency of the $0-4 \mathrm{~m} \mathrm{~s}^{-1}$ class could explain the agreement between RegClaris and ERA-Interim for the SAM withdrawal (Pentad 22). RegZhang shows fewer events of this class; therefore, it presents a delay of 1 pentad for the end of the SAM.

Another wind-based index is MWSI which takes into account the vertical shear of the mean meridional wind over northeastern Brazil (see Fig. 1). Using the same threshold as NCEPNCAR/GAN, which only considers MWSI signal inversion (from negative to positive), the ERA-Interim and simulations show SAM timings that are quite different from NCEP-NCAR/ GAN patterns, particularly for the date of SAM demise. Therefore, Table 2 presents MWSI values obtained with a threshold of $-3 \mathrm{~m} \mathrm{~s}^{-1}$ for the ERAInterim and RegZhang and $-5 \mathrm{~m} \mathrm{~s}^{-1}$ for RegClaris. These values were used because the frequency distribution of MWSI (Fig. 7c) shows that these data sets have a small frequency of positive values, which may explain the difficulty of obtaining results similar to those from NCEP-NCAR/GAN using only signal inversion. Therefore, the need to adapt thresholds suggests that MWSI is not a robust index to be applied to SAM timing. Besides, as noted in Gan et al. (2004) and in Table 2, when comparing the MSWI to the other 2 indices (PrecI and ZWSI), it is clear that MWSI delays the SAM onset and extends its demise. This index 


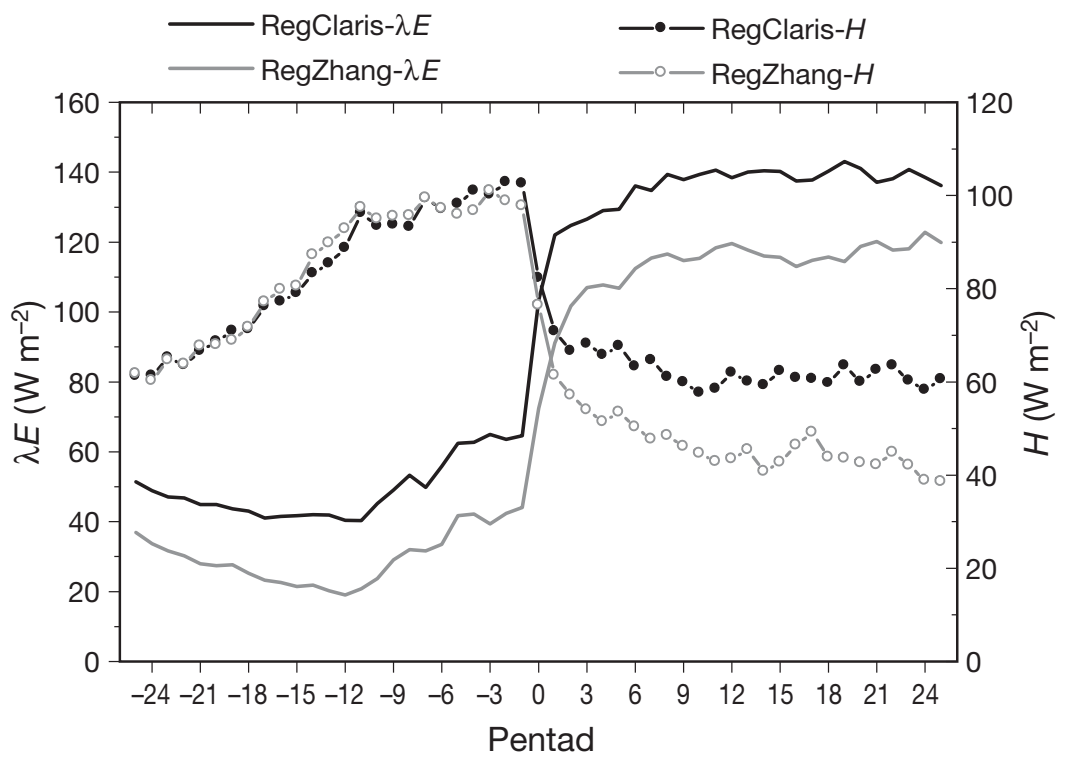

Fig. 8. Time evolution (from 24 pentads before and after SAM onset) of the composites of latent $(\lambda E)$ and sensible $(H)$ heat fluxes simulated by RegClaris and RegZhang. The composites were calculated for the PrecI area shown in Fig. 1

also presents greater variability, as measured by standard deviation, than the others, mainly for demise dates (Table 2).

\section{CONCLUSIONS}

The present study compared 3 simulations performed with RegCM3 over SA. These simulations used the ERA-Interim reanalysis as initial and boundary conditions, considering a domain covering SA. Changes were introduced in the parameters of BATS land-surface model and Grell's convective scheme to reduce the reported RegCM3 rainfall dry bias over tropical SA.

The changes in the convective time period of Grell's scheme, along with modifications in the bottom soil hydraulic conductivity (RegClaris experiment) or the use of deeper soil layers (RegArain experiment) in the BATS land surface model (for the tropical rain forest), resulted in almost the same impact in the simulated rainfall over the AMZ. In this region, RegArian and RegClaris present substantial improvements in prediction of the rainfall rate during the year. These improvements are noted when simulations are compared with gridded analyses (CMAP and CPC rainfall analysis), as well as with in situ data from the AMZ region (rainfall, $\lambda E$ and $H$ fluxes). The proposed RegClaris and RegArian changes enable the model to maintain water in the soil column during the simulation and, consequently, to increase $\lambda E$, the moist static energy (which controls Grell's convective scheme), and the rainfall rate over the AMZ. During the summer season, these changes also resulted in an improvement of the prediction of rainfall rate over the $\mathrm{LPB}$. The latter may be attributed to the increase in low level moisture transport from the AMZ to that area. Although these changes have introduced large improvement in the partition between $H$ and $\lambda E$ fluxes, an overestimation of $R_{\mathrm{n}}$ exists and, consequently, an increase in $H$ and $\lambda E$ in the RegClaris and RegArian experiments. The $R_{\mathrm{n}}$ overestimation is related to considerable activation of Grell's convective scheme, increasing the rainfall rate and diminishing the cloud cover. As a consequence, an increase in the incident solar radiation at surface occurs in the RegClaris and RegArian experiments.

One of the benefits of the improved prediction of rainfall in RegClaris is the better characterization of the SAM system. The errors in the SAM onset and withdrawal dates simulated by RegClaris were very small ( 1 pentad) when compared to those derived from CPC rainfall analyses and CPC/GAN. Applying wind (meridional and zonal) based indices, GAN found that they attained a high correlation with SAM precipitation series, except for a minor tendency of the MWSI to delay the onset of the SAM. However, our application of this index in the ERA-Interim and simulations suggest that it may not be suitable at all times. In fact, the MWSI was unable to reproduce similar SAM timing with simulations or with the ERA-Interim reanalysis. This could be related to the higher horizontal resolution of ERA-Interim $\left(1.5^{\circ}\right)$ and simulations $\left(\sim 0.5^{\circ}\right)$, when compared to the $2.5^{\circ}$ resolution used by GAN, suggesting some dependence of MWSI on grid resolution.

The parameterizations tested suggest the importance of the coupling between surface energy fluxes, atmospheric stability and precipitation, which, during summer, also affect the simulated climate over subtropical SA. The positive impact on water cycle representation suggests the need for in-depth review of the parameters used in the RegCM3 land-surface model for other land-use categories; this will be addressed in the CLARIS-LPB project. 
Acknowledgements. The authors received funding from the European Community's Seventh Framework Programme (FP7/2007-2013) under Grant Agreement No. 212492 (CLARIS LPB - A Europe-South America network for climate change assessment and impact studies in La Plata Basin) and from CAPES/PROCAD 179/2007. R.P.d.R. (CNPq-307519/2008-2) and T.A. (CNPq-300976/2010-0) are also thankful for the support from CNPq and CAPES. We thank the anonymous reviewers for helpful suggestions and comments.

\section{LITERATURE CITED}

Arain AM, Shuttleworth WJ, Yang ZL, Michaud J, Dolman J (1997) Mapping surface-cover parameters using aggregation rules and remotely sensed cover classes. Q J R Meteorol Soc 123:2325-2348

> Arakawa A, Schubert WH (1974) Interactions of a cumulus cloud ensemble with the large-scale environment. I. J Atmos Sci 31:674-701

> Baker IT, Prihodko L, Denning AS, Goulden M, Miller S, da Rocha HR (2008) Seasonal drought stress in the Amazon: reconciling models and observations. J Geophys Res 113: G00B01

Carvalho LMV, Jones C, Liebmann B (2002) Extreme precipitation events in southern South America and largescale convective patterns in South Atlantic Convergence Zone. J Clim 15:2377-2394

> Chou SC, Nunes AMB, Cavalcanti IAF (2000) Extended range forecasts over South America using the regional eta model. J Geophys Res 105:10147-10160

- Cuadra SV, da Rocha RP (2007) Sensitivity of regional climatic simulation over southeastern South America to SST specification during austral summer. Int J Climatol 27:793-804

> da Rocha HR, Goulden ML, Miller SD, Menton MC, Pinto DVOL, de Freitas HC, Figueira EMSA (2004) Seasonality of water and heat fluxes over a tropical forest in eastern Amazonia. Ecol Appl 14:22-32

da Rocha RP, Morales CA, Cuadra SV, Ambrizzi T (2009a) Precipitation diurnal cycle and summer climatology assessment over South America: an evaluation of regional climate model Version 3 simulations. J Geophys Res 114: D10108. doi:10.1029/2008JD010212

da Rocha HR, Manzi AO, Cabral OM, Miller SD and others (2009b) Patterns of water and heat flux across a biome gradient from tropical forest to savanna in Brazil. J Geophys Res 114:G00B12. doi:10.1029/2007JG000640

da Silva M, da Rocha RP, Ynoue RY (2010) Climatic simulations of the eastern Andes low-level jet and its dependency on convective parameterizations. Meteorol Atmos Phys 108:9-27

Dickinson RE, Errico RM, Giorgi F, Bates GT (1989) A regional climate model for the western United States. Clim Change 15:383-422

Dickinson RE, Henderson-Sellers A, Kennedy PJ (1993) Biosphere-atmosphere transfer scheme (BATS) Version $1 \mathrm{E}$ as coupled to the NCAR community climate model. NCAR Tech. Note, NCAR/TN-387, National Center for Atmospheric Research, Boulder, CO

Fan Y, Miguez-Macho G (2010) Potential groundwater contribution to Amazon evapotranspiration. Hydrol Earth Syst Sci 14:2039-2056
Fernandez JPR, Franchito SH, Rao VB (2006) Simulation of summer circulation over South American by two regional climate models. I. Mean climatology. Theor Appl Climatol 86:247-260

Figueroa SN, Satyamurty P, Silva Dias PL (1995) Simulations of the summer circulation over the South American region with an Eta coordinate model. Mon Weather Rev 52:1573-1584

Fu R, Li W (2004) The influence of the land surface on the transition from dry to wet season in Amazonia. Theor Appl Climatol 78:97-110

Gan MA, Kousky VE, Roupelewski CF (2004) The South America monsoon rainfall over West-Central Brazil. J Clim 17:47-66

Gan MA, Rao VB, Moscati MCL (2005) South American monsoon indices. Atmos Sci Lett 6:219-223

Gandu AW, Geisler JE (1991) A primitive equations model study of the effect of topography on the summer circulation over tropical South America. J Atmos Sci 48: 1822-1836

Giorgi F, Marinucci MR (1991) Validation of a regional atmospheric model over Europe: sensitivity of wintertime and summertime simulations to selected physics parameterizations and lower boundary conditions. Q J R Meteorol Soc 117:1171-1206

Giorgi F, Mearns LO (1999) Introduction to special section: regional climate modeling revisited. J Geophys Res 104: 6335-6352

Giorgi F, Jones C, Asrar G (2009) Addressing climate information needs at the regional level: the CORDEX framework. WMO Bulletin 58:175-183

> Grell GA (1993) Prognostic evaluation of assumptions used by cumulus parameterization. Mon Weather Rev 121: 764-787

- Hodnett MG, da Silva LP, da Rocha HR, Sennad RC (1995) Seasonal soil water storage changes beneath central Amazonian rainforest and pasture. J Hydrol (Amst) 170: 233-254

Kiehl JT, Bonan JJ, Boville BA, Briegleb BP, Williamson DL, Rasch PJ (1996) Description of the NCAR community climate model (CCM3). NCAR Tech. Note, NCAR/TN420+STR, National Center for Atmospheric Research, Boulder, CO

Kodama Y (1992) Large-scale common features of subtropical precipitation zones (the Baiu frontal zone, the SPCZ, and the SACZ). I. Characteristics of subtropical frontal zones. J Meteorol Soc Jpn 70:813-836

> Lenters JD, Cook KH (1997) On the origin of the Bolivian high and related circulation features of the South American climate. J Atmos Sci 54:656-677

> Liebmann B, Marengo JA (2001) Interannual variability of the rainy season and rainfall in the Brazilian Amazon basin. J Clim 14:4308-4318

> Marengo JA, Ambrizzi T, da Rocha RP, Alves LM and others (2010) Future change of climate in South America in the late twenty-first century: intercomparison of scenarios from three regional climate models. Clim Dyn 35: 1073-1097

> Negrón Juárez RI, Hodenett MG, Fu R, Goulden ML, von Randow C (2007) Control of dry season evapotranspiration over the Amazonian forest as inferred from observations at a southern Amazon forest site. J Clim 20: 2827-2839

Nepstad DC, de Carvalho CR, Davidson EA, Jipp PH and 
others (1994) The role of deep roots in the hydrological and carbon cycles of Amazonian forests and pastures. Nature 372(Issue 6507):666-669

Nicolini M, Salio P, Katzfey JJ, McGregor JL, Saulo AC (2002) January and July regional climate simulation over South America. J Geophys Res 107:4637-4649

Nunes AMB, Roads JO (2005) Improving regional model simulations with precipitation assimilation. Earth Interact 9:1-44

Nuñez MN, Solman S, Cabré MF (2009) Regional climate change experiments over southern South America. II. Climate change scenarios in the late twenty first century. Clim Dyn 32:1081-1095

Oliveira RS, Dawson TE, Burgess SSO, Nepstad DC (2005) Hydraulic redistribution in three Amazonian trees. Oecologia 145:354-363

Pal JS, Giorgi F, Xunqiang Bi, Elguindi N and others (2007) Regional climate modeling for the developing world: the ICTP RegCM3 and RegCNET. Bull Am Meteorol Soc 88: 1395-1409

Pal JS, Small EE, Eltahir EAB (2000) Simulation of regional scale water and energy budgets: influence of a new moist physics scheme within RegCM. J Geophys Res 105: 29579-29594

Rauscher SA, Seth A, Liebmann B, Qian JH, Camargo SJ (2007) Regional climate model-simulated timing and

Submitted: March 1, 2011; Accepted: September 26, 2011 character of seasonal rains in South America. Mon Weather Rev 135:2642-2657

Seth A, Rauscher S, Qian J, Pal J (2007) RegCM3 regional climatologies for South America using reanalysis and ECHAM global model driving fields. Clim Dyn 28: 461-480

Seth A, Rauscher S, Rojas M, Giannini A, Camargo SJ (2011) Enhanced spring convective barrier for monsoons in a warmer world? Clim Change 104:403-414

Silva VBS, Kousky VE, Shi W, Higgins RW (2007) An improved historical daily precipitation analysis for Brazil. J Hydrometeorol 8:847-861

Simmons A, Uppala S, Dee D, Kobayashi S (2007) ERAInterim: new ECMWF reanalysis products from 1989 onwards. ECMWF Newsletter No. 110, ECMWF, Reading

> Vera CS, Baez J, Douglas M, Emmanuel CB and others (2006) The South American low-level jet experiment. Bull Am Meteorol Soc 87:63-77

> Xie P, Arkin PA (1996) Analyses of global monthly precipitation using gauge observations, satellite estimates, and numerical model predictions. J Clim 9:840-858

Zhang Y, Fu R, Yu H, Dickinson RE, Juarez RN, Chin M, Wang $H$ (2008) A regional climate model study of how biomass burning aerosol impacts land-atmosphere interactions over the Amazon. J Geophys Res 113:D14S15. doi:10.1029/2007JD009449

Proofs received from author(s): January 27, 2012 